\title{
Level of mesothelin expression can indicate the prognosis of malignant pleural mesothelioma
}

\author{
Feifei Feng, Huanan Zhang, Ying Zhang, Hui Wang \\ Department of Respiratory Medicine, The Second Hospital, Cheeloo College of Medicine, Shandong University, Jinan, China \\ Contributions: (I) Conception and design: F Feng, H Wang; (II) Administrative support: H Wang, H Zhang; (III) Provision of study materials or \\ patients: F Feng, H Zhang, Y Zhang; (IV) Collection and assembly of data: F Feng, H Wang; (V) Data analysis and interpretation: F Feng, H Wang; \\ (VI) Manuscript writing: All authors; (VII) Final approval of manuscript: All authors. \\ Correspondence to: Dr. Hui Wang. Department of Respiratory Medicine, The Second Hospital, Cheeloo College of Medicine, Shandong University, \\ 247 Beiyuan Street, Jinan 250033, China. Email: 570864721@qq.com.
}

\begin{abstract}
Background: Malignant pleural mesothelioma (MPM) is a fatal, treatment-resistant tumor. The median survival of MPM is 9-12 months and its early prognostic markers remains uncertain. The objective of this study was to determine that the level of mesothelin expression can be as a predictor of prognosis in MPM patients.

Methods: Level of mesothelin expression was detected in 38 MPM tissue specimens by immunohistochemistry analysis. The relationship of MPM prognosis and mesothelin expression was evaluated by univariate and multivariate Cox regression, Kaplan-Meier survival curves.

Results: High level of mesothelin expression was significantly associated with non-epithelioid type of MPM and smoking. Meanwhile, higher level of mesothelin expression indicated a shorter total survival.

Conclusions: The present study suggested that mesothelin is a dependent prognostic factor in MPM patients and might be a novel potential target for immunotherapy in MPM.
\end{abstract}

Keywords: Malignant pleural mesothelioma (MPM); mesothelin; prognosis; survival

Submitted Sep 26, 2019. Accepted for publication Feb 05, 2020.

doi: $10.21037 /$ tcr-19-2027

View this article at: http://dx.doi.org/10.21037/tcr-19-2027

\section{Introduction}

Malignant pleural mesothelioma (MPM) is an aggressive form of cancer that is caused by exposure to asbestos, which incidence is increasing worldwide (1). The global mesothelioma burden is estimated to be in the range of 36,300 to 38,400 annual deaths, according to the database of The World Health Organization (WHO) (1994 to 2014) (2). MPM has very poor prognosis due to its chemoresistance and radioresistance, with the median survival time (MST) of 9-12 months $(3,4)$. MPM is difficult to early diagnosis resulting in few surgery opportunities for most patients with MPM. Therefore, early detection and diagnosis of MPM may help to increase its surgery opportunity and improve its prognosis.

In nowadays, several molecules, including estrogen receptors (5), matrix metalloproteinase 2 and $9(6,7)$ have been reported as biomarkers for MPM. However, these factors were not available for early diagnosis due to lack of specificity. Therefore, it is very valuable to find a new prognostic factor for early diagnosis of MPM. Mesothelin is a glycosylphosphatidylinositol (GPI)-anchored cellsurface protein, which level of expression is low in normal tissues and high in a variety of malignant tumors, such as ovarian cancer, pancreatic cancer (8), and mesotheliomas (9). It has been reported that mesothelin can be as a marker of neoplastic progression and immunotherapy target in gastroesophageal cancer (10). However, the prognostic value of soluble mesothelin in malignant pleural mesothelioma remains unclear.

In the present study, the mesothelin expression profile in MPM tissues was analyzed using immunohistochemistry (IHC) staining method, and its prognostic value was evaluated using statistic methods. 
Table 1 Clinical characteristics of the MPM patients with expression of mesothelin

\begin{tabular}{lccc}
\hline Mesothelin expression & Low & High & P value \\
\hline Cases & 26 & 12 & \\
Gender & & & 1.000 \\
Male & 20 & 10 & \\
Female & 6 & 2 & \\
Age & & & 0.694 \\
$<60$ & 6 & 4 & \\
$\geq 60$ & 20 & 8 & \\
Smoking status & & & $0.030^{\star}$ \\
Non-smoker & 12 & 1 & \\
Smoker & 14 & 11 & \\
Stage & & & 1.000 \\
Early (I-II) & 11 & 5 & \\
Late (III-IV) & 15 & 7 & \\
Pathological type & & & \\
Epithelioid & 24 & 5 & \\
Non-epithelioid & 2 & 7 & \\
\hline
\end{tabular}

*indicates statistical significant difference.

\section{Methods}

\section{Patients and specimens}

Thirty-eight patients with diagnosed MPM in the Second Hospital of Shandong University between May 2007 and December 2014 were enrolled, and followed up to August 2015. Tumor grade were determined according to the staging of International Mesothelioma Interest group (IMIG). This study was approved by the Ethics Committee of the Second Hospital of Shandong University (Permit Number: 20140195). The trial was conducted in accordance with the Declaration of Helsinki (as revised in 2013). Because of the retrospective nature of the research, the requirement for informed consent was waived.

\section{Immunobistochemistry}

Paraffin-embedded MPM specimens were cut into $5 \mathrm{~mm}$ sections for immunohistochemistry analysis. Sections were deparaffinized in xylene and then rehydrated with ethanol. For antigen retrieval, the specimens were incubated in a microwave oven $\left(95^{\circ} \mathrm{C}\right)$ in ethylene diamine tetraacetic acid (EDTA) (ZLI-9068, Zhongshan Golden Bridge Bio-technology, China) for $15 \mathrm{~min}$. After natural cooling, endogenous peroxidase activity was blocked with $3 \%$ hydrogen peroxide for $15 \mathrm{~min}$ at room temperature. Then, sections were washed with phosphate buffer saline (PBS) for $5 \mathrm{~min}$. Nonspecific antibody binding was blocked with goat serum (ZLI-9022, Zhongshan Golden Bridge Biotechnology, China) for $10 \mathrm{~min}$. Subsequently, the specimens were incubated with rabbit polyclonal antimesothelin antibody $\left(1: 200\right.$, ab96869, abcam, UK) at $4{ }^{\circ} \mathrm{C}$ for overnight. Rewarming at $37^{\circ} \mathrm{C}$ and then washed with PBS, the specimens were incubated with goat anti-rabbit secondary antibody conjugated with horseradish peroxidase (HRP) (PV-9000, Zhongshan Golden Bridge Bio-technology, China). Counter-stained with hematoxylin, the sections were rinsed, dehydrated and covered.

\section{Immunobistochemical scoring}

The randomly selected slides were evaluated by two independent pathologists who were blinded to the clinical status of patients. The intensity of mesothelin expression was scored from 0 to $3+$ according to the percentage of positive cells: 0 (trace or $1-25 \%$ positive expression), $1+(26-50 \%$ positive expression $), 2+(51-75 \%$ positive expression), $3+$ (76-100\% positive expression). Scores of 0 and $1+$ were classified as low expression and scores of $2+$ and $3+$ were classified as high expression.

\section{Statistical analysis}

SPSS 17.0software (SPSS Inc., Chicago, IL) was used to perform statistical analysis. The correlation between mesothelin expression and pathologic and staging features was analyzed by Fisher's exact test. Survival information was plotted using the Kaplan-Meier curves and the log rank test. Univariate and multivariate analysis of prognostic factors was performed using Cox's proportional hazards regression model. $\mathrm{P}<0.05$ was used as the cut-off value and considered statistically significant.

\section{Results}

\section{Mesothelin expression and patient characteristics in MPM}

A total of 38 patients were enrolled in the present study, including 30 males and 8 females. Median patient age was $66.11 \pm 9.45$ years. Twenty-six cases $(68 \%)$ had a smoking history. 29 (76\%) patients were epithelioid type and the others were non-epithelioid type, which included sarcomatoid and biphasic. More details were shown in Table 1 . 

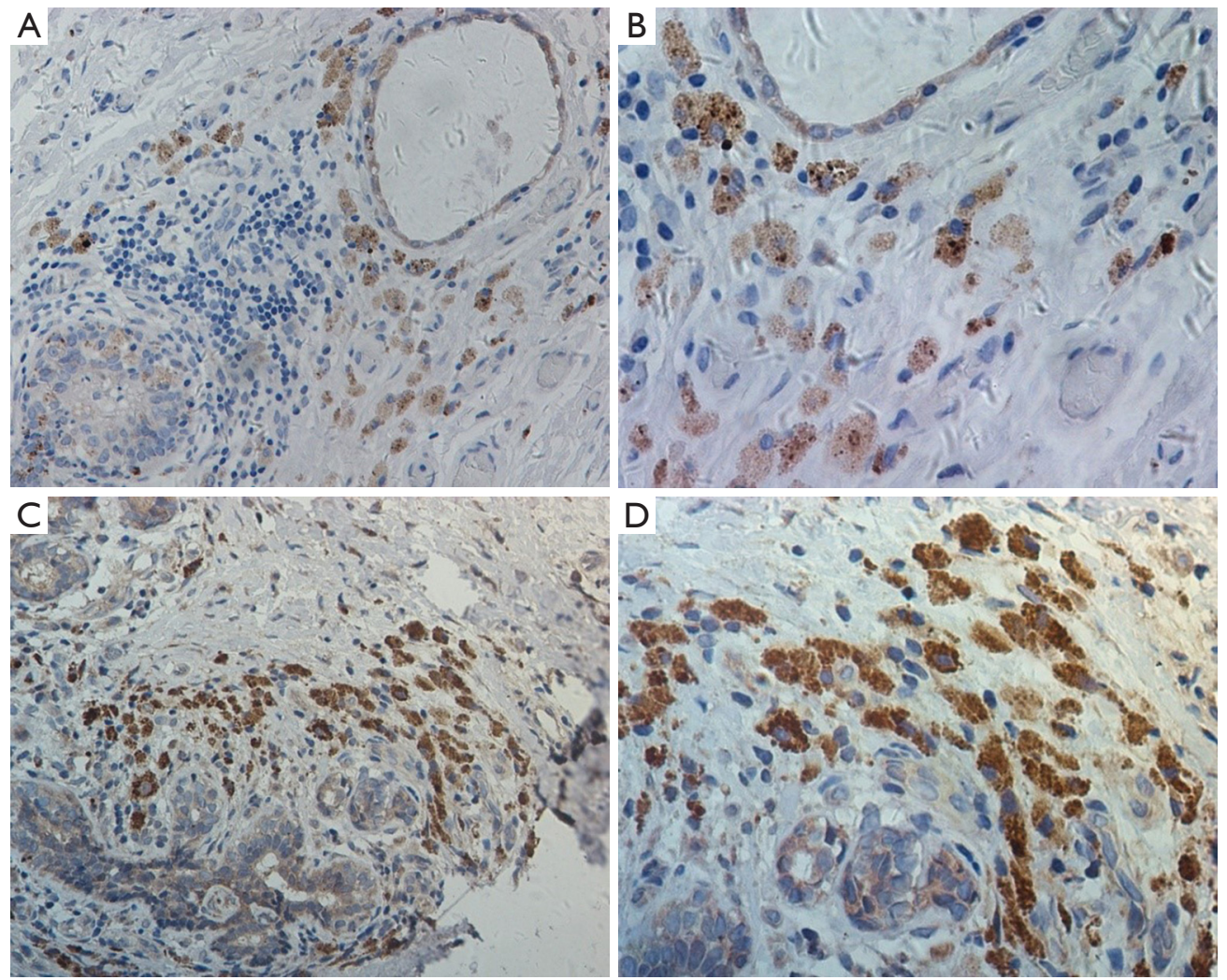

Figure 1 Immunohistochemical results of mesothelin expression in MPM patients. Low-level expression of mesothelin are shown in A (magnification, 100x) and B (magnification, 200x), and high-level expression are shown in C (magnification, 100x) and D (magnification, $200 \times)$.

The high-level expression of mesothelin were significantly associated with higher in smokers $(\mathrm{P}=0.030)$ and non-epithelioid type $(\mathrm{P}=0.002)$. There is no significant difference of mesothelin expression in other clinic characteristics. Representative pictures for mesothelin staining are shown in Figure 1.

\section{Relationship between overall survival of MPM and patient characteristics or mesothelin expression}

To determine the prognostic factors of MPM, the overall survival (OS) of MPM patients was analyzed based on IHC between patient characteristics and mesothelin expression respectively (Figure 2).

The smokers have shorter OS $(9.63 \pm 1.11$ months $)$ than those who do not smoke $(17.02 \pm 1.47$ months) $(\mathrm{P}=0.014$, Figure $2 A)$. The other significant difference of OS was between epithelioid type $(14.62 \pm 1.32$ months $)$ and nonepithelioid type $(6.17 \pm 1.37$ months $)(\mathrm{P}<0.001$, Figure $2 E)$. These results are in accord with the established knowledge.
It is worth noting that the patients with high expression $(7.47 \pm 1.48$ months) of mesothelin had shorter OS than those with low expression $(14.72 \pm 1.30$ months $)(\mathrm{P}=0.005$ Figure $2 F)$. However, no significant difference of OS was observed in subgroup of gender, age and stage (Figure $2 B, C, D$ ).

To determine which factors had the influence on the MPM patients' overall survival, various possible predictive factors were engaged in univariate analysis (Table 2). The results indicated that smoking status $(\mathrm{P}=0.023)$, pathological type $(\mathrm{P}<0.001)$, and mesothelin expression $(\mathrm{P}=0.009)$ had significant effects on OS, which confirmed to the results of Kaplan-Meier overall survival curve. By multivariate Cox analysis, it was confirmed that non-epithelioid type $(\mathrm{P}=0.037)$ and high mesothelin expression $(\mathrm{P}=0.038)$ were the independent negative prognostic factors of OS for patients with MPM (Table 3).

\section{Discussion and conclusion}

High-level expression of mesothelin has been reported 
A

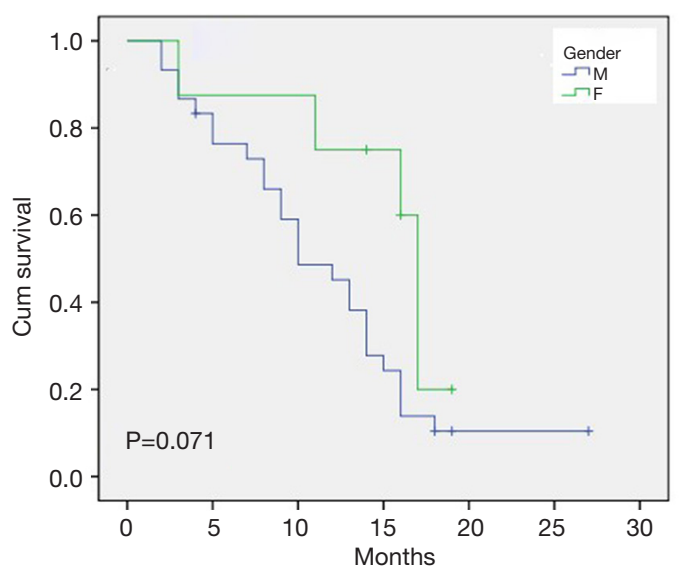

C

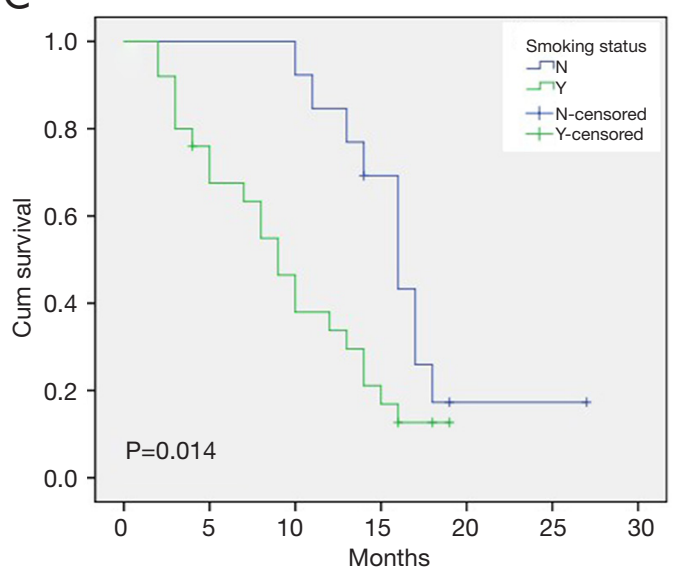

E

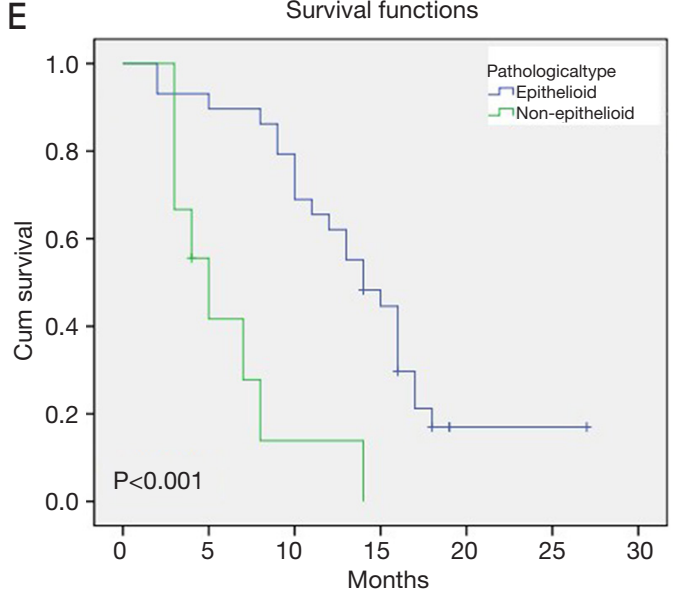

B

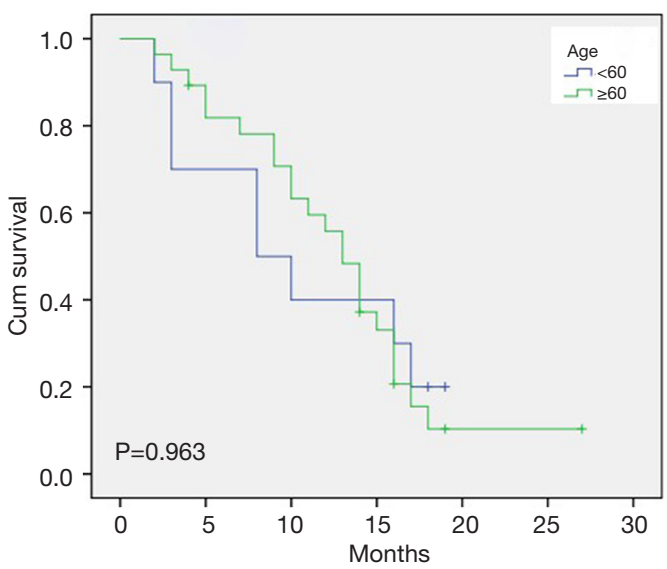

D

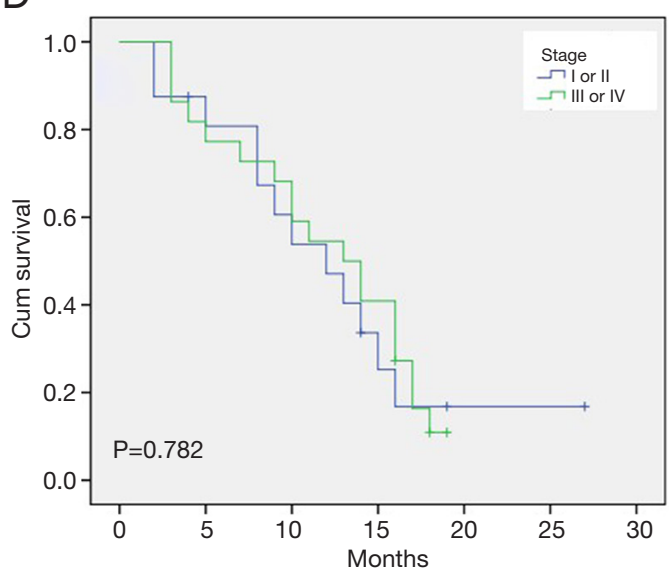

F

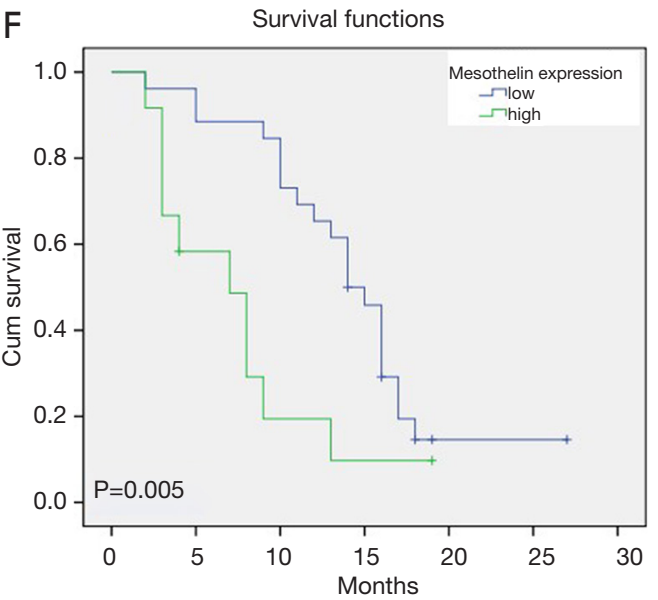

Figure 2 Relationship between overall survival of MPM and patient characteristics or mesothelin expression. Kaplan-Meier survival curves by the status of (A) gender, (B) age, (C) smoking status, (D) stage, (E) pathological type, (F) mesothelin expression. 
Table 2 Univariate analysis of overall survival in 38 patients with MPM

\begin{tabular}{lccc}
\hline Patient characteristics & Overall survival hazard radio & $95 \% \mathrm{Cl}$ & $\mathrm{P}$ value \\
\hline Gender: male vs. female & 2.284 & $0.870-6.001$ & 0.094 \\
Age: $<60$ vs. $\geq 60$ & 0.982 & $0.435-2.216$ \\
Smoking status: no vs. yes & 0.408 & $0.189-0.882$ & 0.065 \\
Stage: I-II vs. III-IV & 1.104 & $0.553-2.286$ & 0.790 \\
Pathological type: Epithelioid vs. non- epithelioid & 0.192 & $0.076-0.482$ & $0.000^{*}$ \\
Mesothelin: low vs. high & 0.348 & $0.158-0.765$ \\
\hline
\end{tabular}

*indicates statistical significant difference.

Table 3 Multivariate analysis of overall survival in 38 patients with MPM

\begin{tabular}{lccc}
\hline Patient characteristics & Overall survival hazard radio & $95 \% \mathrm{Cl}$ & $\mathrm{P}$ value \\
\hline Gender: male vs. female & 1.980 & $0.631-6.217$ & 0.242 \\
Age: $<60$ vs. $\geq 60$ & 0.821 & $0.347-1.940$ \\
Smoking status: no vs. yes & 0.630 & $0.244-1.626$ \\
Stage: I-II vs. III-IV & 1.067 & $0.494-2.304$ & 0.911 \\
Pathological type: Epithelioid vs. non- epithelioid & 0.330 & $0.116-0.935$ & 0.869 \\
Mesothelin: low vs. high & 0.377 & $0.150-0.947$ & $0.038^{\star}$ \\
\hline
\end{tabular}

*indicates statistical significant difference.

in several type of malignant tumors including MPM, lung, ovarian, pancreatic and esophageal cancer (8-10). However, its prognostic value in patients with MPM was still unknown. In the present study, the relationship between mesothelin expression and prognosis of MPM was investigated.

Mesothelin is a $40-\mathrm{kDa}$ glycosyl-phosphatidylinositollinked cell-surface glycoprotein (11). It has been reported that it might play an important role in cell adhesion and metastasis in ovarian cancers (8) and be a potential marker of neoplastic progression $(12,13)$. In human lung cancer and mesothelioma cells, overexpression of mesothelin can promote epithelialto-mesenchymal transition and tumorigenicity, then leads to tumor growth and metastasis (9).

In MPM, it has been known that male gender, age and asbestos exposure are considered as poor prognostic factors $(14,15)$. Perspective from pathology in MPM, nonepithelioid type may have a worse prognosis and a longer history of asbestos exposure of higher intensity $(16,17)$. In the present study, it has been showed that the high-level expression of mesothelin was prevalent in the smokers and non-epithelioid type. Meanwhile, the patients with high mesothelin expression showed a significantly lower OS than those with low expression. Multivariate analysis determined that mesothelin was a independent negative prognostic factor in OS of patients. As the mesothelin expression could be easily detected by immunohistochemistry, it can be served as a promising tumor biomarker for prognosis in MPM patients.

Though the previous study presented the survival advantage in female MPM patients compared to male (17). There is no significant difference of OS between different genders in the present results. Moreover, patients in early stage have not showed longer survival duration than those in late stage. The reason might be the small number of enrolled cases. Therefore, a multi-institutional study which should enroll more MPM cases will be performed.

Several studies showed that mesothelin might be a novel target for immunotherapy among several cancers (18). The treatment based on mesothelin antibody has been studied in ovarian, pancreatic cancer (8), gastroesophageal cancer (10), glioblastoma (11) and malignant mesothelioma (19). The present study confirmed that high-level expression of mesothelin in MPM predicted the poor prognosis and act 
as a $\mathrm{n}$ independent factor. Therefore, the down-regulation expression of mesothelin in MPM would be considered as a novel target therapy for MPM.

\section{Acknowledgments}

We thanked language edit performed by professional editors at Editage.

Funding: The study was financially supported by the Natural Science Foundation of China (Grant No.81473485), the Natural Science Foundation of China (Grant No.81973630) and Jinan Science and Technology Development Program (Grant No. 201805067).

\section{Footnote}

Conflicts of Interest: All authors have completed the ICMJE uniform disclosure form (available at http://dx.doi. org/10.21037/tcr-19-2027). The authors have no conflicts of interest to declare.

Ethical Statement: The authors are accountable for all aspects of the work in ensuring that questions related to the accuracy or integrity of any part of the work are appropriately investigated and resolved. The present experiment was approved by the Scientific Research Ethics Committee of the Second Hospital of Shandong University (Permit Number: 20140195). The trial was conducted in accordance with the Declaration of Helsinki (as revised in 2013). Because of the retrospective nature of the research, the requirement for informed consent was waived.

Open Access Statement: This is an Open Access article distributed in accordance with the Creative Commons Attribution-NonCommercial-NoDerivs 4.0 International License (CC BY-NC-ND 4.0), which permits the noncommercial replication and distribution of the article with the strict proviso that no changes or edits are made and the original work is properly cited (including links to both the formal publication through the relevant DOI and the license). See: https://creativecommons.org/licenses/by-nc-nd/4.0/.

\section{References}

1. American Cancer Society. Malignant mesothelioma. Washington, 2015. Available online: http://www.cancer. org/acs/groups/cid/documents/webcontent/003119-pdf (accessed 22 Feb 2016).
2. Odgerel CO, Takahashi K, Sorahan T, et al. Estimation of the global burden of mesothelioma deaths from incomplete national mortality data. Occup Environ Med 2017;74:851-8.

3. Kondola S, Manners D, Nowak AK. Malignant pleural mesothelioma: an update on diagnosis and treatment options. Ther Adv Respir Dis 2016;10:275-88.

4. Bille A, Krug LM, Woo KM, et al. Contemporary Analysis of Prognostic Factors in Patients with Unresectable Malignant Pleural Mesothelioma. J Thorac Oncol 2016;11:249-55.

5. Pillai K, Pourgholami MH, Chua TC, et al. Oestrogen receptors are prognostic factors in malignant peritoneal mesothelioma. J Cancer Res Clin Oncol 2013;139:987-94.

6. Štrbac D, Goričar K, Dolžan V, et al. Evaluation of Matrix Metalloproteinase 9 Serum Concentration as a Biomarker in Malignant Mesothelioma. Dis Markers 2019;2019:1242964.

7. Muscella A, Cossa LG, Vetrugno C, et al. Adenosine diphosphate regulates MMP2 and MMP9 activity in malignant mesothelioma cells. Ann N Y Acad Sci 2018;1431:72-84.

8. Lamberts LE, Menke-van der Houven van Oordt CW, ter Weele EJ, et al. ImmunoPET with Anti-Mesothelin Antibody in Patients with Pancreatic and Ovarian Cancer before Anti-Mesothelin Antibody-Drug Conjugate Treatment. Clin Cancer Res 2016;22:1642-52.

9. He X, Wang L, Riedel H, et al. Mesothelin promotes epithelial-to-mesenchymal transition and tumorigenicity of human lung cancer and mesothelioma cells. Mol Cancer 2017;16:63.

10. Illei PB, Alewine C, Zahurak M, et al. Mesothelin Expression in Advanced Gastroesophageal Cancer Represents a Novel Target for Immunotherapy. Appl Immunohistochem Mol Morphol 2016;24:246-52.

11. Liu Z, Rao M, Poiret T, et al. Mesothelin as a novel biomarker and immunotherapeutic target in human glioblastoma. Oncotarget 2017;8:80208-22.

12. Anish Thomas, Yuanbin Chen, Seth M. Steinberg, et al. High mesothelin expression in advanced lung adenocarcinoma is associated with KRAS mutations and a poor prognosis. Oncotarget 2015;6:11694-703.

13. Bayoglu IV, Kucukzeybek BB, Kucukzeybek Y, et al. Prognostic value of mesothelin expression in patients with triple negative and HER2-positive breast cancers. Biomed Pharmacother 2015;70:190-5.

14. Davidson B. Prognostic factors in malignant pleural mesothelioma. Hum Pathol 2015;46:789-804. 
15. Billé A, Krug LM, Woo KM, et al. Contemporary Analysis of Prognostic Factors in Patients with Unresectable Malignant Pleural Mesothelioma. J Thorac Oncol 2016;11:249-55.

16. Pass HI, Goparaju C, Espin-Garcia O, et al. Plasma Biomarker Enrichment of Clinical Prognostic Indices in Malignant Pleural Mesothelioma. J Thorac Oncol 2016;11:900-9.

17. Amin W, Linkov F, Landsittel DP, et al. Factors influencing malignant mesothelioma survival: a retrospective review of

Cite this article as: Feng F, Zhang H, Zhang Y, Wang H. Level of mesothelin expression can indicate the prognosis of malignant pleural mesothelioma. Transl Cancer Res 2020;9(12):7479-7485. doi: 10.21037/tcr-19-2027 the National Mesothelioma Virtual Bank cohort. Version 3. F1000Res 2018;7:1184.

18. Zhao XY, Subramanyam B, Sarapa N, et al. Novel Antibody Therapeutics Targeting Mesothelin In Solid Tumors. Clin Cancer Drugs 2016;3:76-86.

19. Zhang J, Khanna S, Jiang Q, et al. Efficacy of Antimesothelin Immunotoxin RG7787 plus Nab-Paclitaxel against Mesothelioma Patient-Derived Xenografts and Mesothelin as a Biomarker of Tumor Response. Clin Cancer Res 2017;23:1564-74. 